\title{
Forgotten coindex of some non-toxic dendrimers structure used in targeted drug delivery
}

https://doi.org/10.1515/mgmc-2021-0004

received October 20, 2020; accepted November 22, 2020

Abstract: In recent past, porphyrin-based dendrimers have gained great attraction due to their usefulness in nano devices and photo-dynamic therapy. New technologies based upon nano-materials or dendrimers have potential to overcome the problems due to conventional drug delivery, like toxicity, poor solubility and poor release pattern of drugs. The chemical and physical properties of these highly branched nanometer sized dendrimers depend on their structure. In chemical network theory, various topological indices are used to predict chemical properties of molecules(dendrimers). Among many useful topological descriptors forgotten coindex is relatively less explored but is found very useful in material engineering, pharmaceutical and chemical industries. In this article, we consider some special dendrimers, like poly(propyl) ether imine, porphyrin, and zinc-porphyrin, and nanostars like $D_{1}[k]$ and $D_{2}[k]$ and compute forgotten coindex for these important structures.

Keywords: topological descriptors, forgotten coindex, nanostar dendrimers, poly(propyl) ether imine, porphyrin and zinc-porphyrin dendrimers

\section{Introduction}

Average cost of new drugs is rising faster than the inflation rate. The main reason for this rapid increase in prices of drugs is due to expenditures on $R \& D$ to

\footnotetext{
* Corresponding author: Yasir Ali, College of Electrical and Mechanical Engineering, National University of Sciences and Technology, Islamabad, Pakistan, e-mail: yali@ceme.nust.edu.pk

Zainab Bibi: School of Natural Sciences, National University of Sciences and Technology, Islamabad, Pakistan, e-mail: Zainabbangash398@gmail.com

Quanita Kiran: School Electrical Engineering and Computer Science, National University of Sciences and Technology, Sector H-12, Islamabad, Pakistan, e-mail: quanita.kiran@seecs.edu.pk
}

analyze the side effects and performance of the drugs. Other reasons include appropriate apparatus, human resources, systems of transportation etc. All such aspects are studied under the umbrella of "drug delivery" and "cheminformatics". Drug delivery is a vast field covering technologies, formulations, approaches, and means of transporting a pharmaceutical compound in the human body to achieve its desired therapeutic effect. Targeted delivery is an important branch of drug delivery. In targeted drug delivery we ensure that the drug is active only in the concerned area or portion of the body, see Mitragotri et al. (2014). Dendrimers and polymers play a pivotal role in the molecular toolbox for drug delivery as they are being used in anticancer drug targeting, for instance see Gonzaga et al. (2018), Lee et al. (2015), and Yang (2016). Suitably modified dendrimers have important applications in transdermal drug delivery that is an alternative to the oral administration. It allows direct delivery in the form of a patch or ointment through the skin into the tissue, see Guo et al. (2014) and Sun et al. (2012). Prophyrin dendrimers play a vital role in photodynamic therapy and nano devices due to their unique property of transferring their excitation energy on commotion by light, for instance see: Ethirajan et al. (2011), Jeong et al. (2012), and O'Connor et al. (2009). Different structures and shapes of dendrimers are very useful in the study of transfection, chemotherapy, and gene therapy, for detailed studies see: Bielinska et al. (1999), Jain et al. (2010), Lakshminarayanan et al. (2013), Xiong et al. (2018), and Yang et al. (2015). Authors of these papers explore bio-distribution and toxicity of dendrimers (and polymers) with dependency on their structures. It is clear from the literature, especially from Gao et al. (2020a), Imran et al. (2014), Jeong et al. (2012), and Sadamoto et al. (1996), that chemical properties of drugs are closely related to their molecular structures. To explore such relations a new field "cheminformatics" is emerging as a combination of chemistry, information science and mathematics. It mainly deals with the study and implementation of topological indices and correlation of their physic-chemical properties like 
boiling/melting point, strain energy and stability etc, to the industries especially to the pharmaceutical industry see Imran et al. (2014). A comprehensive survey of many degree-based topological indices and related chemical is given by (Gutman, 2013) and for very recent studies readers are referred to (Gao et al., 2020b, 2020c). Here we are highlighting some important studies that establish the physic-chemical properties or reactivity of molecular structures. The Zagreb indices (first and second) of a graph are one of the most studied vertex degree based topological indices. Gutman and Trinajstić (1972) explored that the total $\pi$-electron energy is dependent upon its structure. They also established the formulas to compute the total $\pi$-electron energy. The mathematical representation of these studies depend upon the graphs. Let $G(V(G), E(G)$ ) be a connected graph and $V(G)$ and $E(G)$ denote the vertex and edge sets, respectively. Further note that the order and size of $G$ are given by $|V(G)|=n$ and $|E(G)|$, respectively. Note that an edge $e=u v \in E(G)$ implies that $u$ and $v$ are adjacent. On the other hand uw $\notin E(G)$ shows that vertices $u$ and $w$ are not adjacent. Moreover $d_{u}$ denotes the degree of $u \in V(G)($ or $u \in G)$ and $N_{u}$ denotes the number of vertices not adjacent to $u \in G$, mathematically,

$$
N_{u}=|\{w \mid u w \notin E(G)\}|, \quad \text { for } u \in G .
$$

With the help of the notation we can write the formulas presented in Gutman and Trinajstić (1972) in mathematical form. The formulas given by Gutman and Trinajstić (1972) to approximate the total $\pi$ - electron energy are known as the first and second Zagreb indices and defined respectively by:

$$
M_{1}=\sum_{u v \in E(G)}\left[d_{u}+d_{v}\right]
$$

and

$$
M_{2}=\sum_{u v E(G)} d_{u} \cdot d_{v}
$$

where $d_{u}$ denotes the degree of a vertex $u$. Recent studies on Zagreb indices and distance based indices may be found in Liu et al. (2019), Siddiqui (2020), and Xu et al. (2019). In 1998, ABC-index were introduced by Estrada et al. (1998). Later in Estrada (2008) and Gutman et al. (2012) many important properties of $\mathrm{ABC}$-index are explored to establish that there is a strong correlation between the thermodynamical properties of alkanes and their $\mathrm{ABC}$ index.

Another vertex-degree based topological index that was introduced in Gutman and Trinajstić (1972), has been explored after for more than forty years by Furtula and Gutman (2015). This index is defined as:

$$
F(G)=\sum_{u v \in E(G)}\left[d_{u}^{2}+d_{v}^{2}\right]
$$

It is known as " forgotten index" and is denoted by "F-index". F-index is known to have useful in chemical testing and it is studied for some important drug molecular structures, details can be seen in Gao et al. (2016) and Sun et al. (2014). Study of F-index is extended to infinite family of nanostar dendrimers by De and Abu Nayeem (2016) and De et al. (2016) defined coindex version of F-index as:

$$
\bar{F}=\sum_{u v E E(G)}\left[d_{u}^{2}+d_{v}^{2}\right] .
$$

De and his coauthors in De et al. (2016b) studied the applicability of F-coindex by using a dataset of octane isomers that is available for boiling/melting point, entropy, density, heat capacities, heat of vaporization and total surface area, etc. They found strong correlation between F-coindex values of octane isomers and the logarithm of the octanol-water partition coefficient $(P)$. They also shown that the correlation of $\log P$ with F-coindex is stronger than its correlation with the first Zagreb index and with F-index. More useful studies of F-coindex on different structures that are used frequently in material engineering, pharmaceutical and chemical, such as nanotubes, nanotorus and graphene sheet can be found in Amin and Abu Nayeem (2017), Bashir et al. (2017), Berhe and Wang (2019), De (2018), Diudea et al. (2010), and Shao et al. (2018). Even though forgotten topological indices and coindices have tremendous industrial and pharmaceutical interests but explorations of F-index and coindex are still limited. With this in mind we explore some properties of zinc-porphyrin, nanostar, poly(propyl) ether imine, porphyrin dendrimers in connection to F-coindex.

First of all we present an alternative form of F-coindex given by Eq. 5, with the help of the following property of graphs.

\section{Lemma 1.1}

Let $\mathrm{G}$ be any graph with $\mathrm{n}$ vertices and $\mathrm{u} \notin \mathrm{G}$ such that degree of $u$ is $d_{u}$. Then, the number of non-adjacent vertices to $u$ is given by:

$$
N_{u}=n-1-d_{u} .
$$

Proof. There are $n-1$ vertices other than $u$ and $u$ is adjacent to $d_{u}$ vertices. Thus there are $n-1-d_{u}$ vertices that are not adjacent to $u$. 
Lemma 1.1 represents the relationship between nonadjacent vertices and order $n$ of a graph.

\section{F-coindex an alternative formula}

Here we present a new form of F-coindex that makes computations of F-coindex simpler than the formula given by Eq. 5 .

\section{Theorem 2.1}

Let $\mathrm{G}$ be any graph the formula given by Eq. 5 of F-coindex of $\mathrm{G}$ is equivalently expressed in the following ways:

$$
\bar{F}(G)=\sum_{u \in G} d_{u}^{2} \times N_{u}
$$

Also,

$$
\bar{F}(G)=\sum_{u \in G}\left[(n-1) d_{u}^{2}-d_{u}^{3}\right]
$$

Proof. We know that the F-coindex for any graph $G$ is given by:

$$
\bar{F}(G)=\sum_{u v \notin E(G)}\left[d_{u}^{2}+d_{v}^{2}\right] .
$$

For any vertex $u$, the term $d_{u}^{2}$ appears in F-coindex for each non-adjacent vertex to $u$. This means that $d_{u}^{2}$ appears $N_{u}$ times. The same is true for each vertex. Thus:

$$
\bar{F}(G)=\sum_{u \in G} d_{u}^{2} \times N_{u}=\sum_{u \in G}\left[(n-1) d_{u}^{2}-d_{u}^{3}\right],
$$

by using Eq. 6.

\section{The F-coindex of nanostar dendrimers}

This section includes the study of F-coindex for some important families of nanostars dendrimers. First we, very breifly, explain the structures of these nanostars and then we compute their F-coindex.

\subsection{F-coindex of type-I nanostar dendrimers $\left(D_{1}[k]\right)$}

The structures of type-I nanostar dendrimer, for $k=1$ and $k=2$, are given in Figure 1. It can be seen that order and size of $D_{1}[1]$ are 24 and 27, respectively, where as of $D_{1}$ [2] its 60 and 67. Generally we can obtain order of $D_{1}[k]$ by $2^{n}(18)-12$ and size of $D_{1}[k]$ is obtained by $27+42(n-1)$.

Following theorem gives us information about F-coindex of $D_{1}[k]$ nanostar dendrimers as shown in Figure 1.

\section{Theorem 3.1}

The F-coindex of nanostar dendrimers $D_{1}[k]$ is denoted by $\overline{\mathrm{F}}\left(\mathrm{D}_{1}[\mathrm{k}]\right)$ and is given as follows:

$$
\bar{F}\left(D_{1}[k]\right)=1836\left(4^{k}\right)-2988\left(2^{k}\right)+1224, \quad \text { for } k \geq 1 \text {. }
$$

Proof. As can be seen from Figure 1, growth of nanostar dendrimers $D_{1}[k]$ is symmetrical. We can use this symmetry to compute F-coindex of $D_{1}[k]$ just by labeling of single branch of $D_{1}[k]$. On the basis of frequency, non-adjacency and degree we select two representative vertices say $u$ and $v$, for the central hexagon. Other representatives for the branch are labeled as: $a_{i}, b_{i}, c_{i}, d_{i}$ where $1 \leq i \leq n$. Note that $c_{i}$ for $1 \leq i \leq n-1$ and $c_{n}$ would be different. The information required for computation of F-coindex for all these representatives are given in the Table 1.

Now with the help of Table 1 and formula given by Eq. 7 we can write the F-coindex of $D_{1}[1]$ as follows:

$$
\begin{aligned}
\bar{F}\left(D_{1}[1]\right)= & 2^{2}(3) 21+3^{2}(3) 20+3^{2}(3) 20 \\
& +2^{2}(6) 21+2^{2}(3) 21+2^{2}(3) 21=2592 .
\end{aligned}
$$

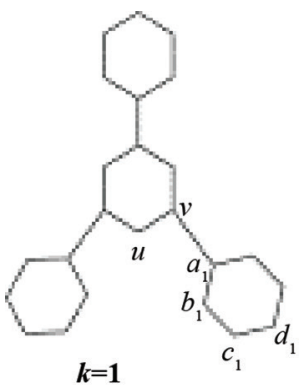

$k=1$

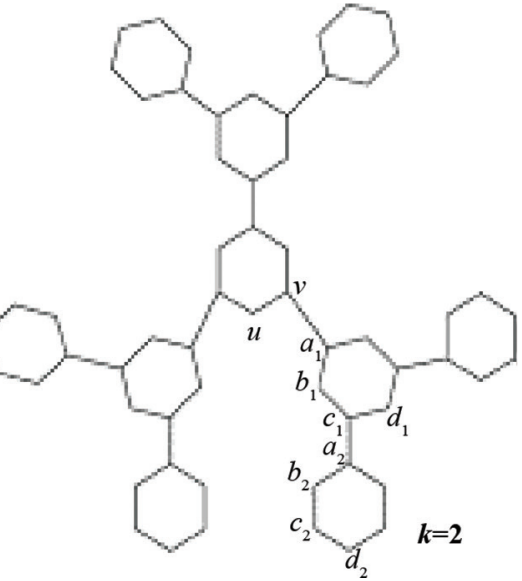

Figure 1: $D_{1}[k]$ nanostar dendrimers for $k=1$ and 2 . 
The F-coindex of $D_{1}[k]$ for $n \geq 2$ can be written as follows:

$$
\begin{aligned}
\bar{F}\left(D_{1}[k]\right)= & 4 \cdot\left(2^{k}(18)-15\right) \cdot\left(3+6 \cdot\left(\sum_{i=1}^{k} 2^{i-1}\right)+3 \cdot\left(\sum_{i=1}^{k} 2^{i-1}\right)+6 \cdot 2^{k-1}\right) \\
& +9 \cdot\left(2^{k}(18)-16\right)\left(3+3 \cdot\left(\sum_{i=1}^{k} 2^{i-1}\right)+6 \cdot\left(\sum_{i=1}^{k-1} 2^{i-1}\right)\right)
\end{aligned}
$$

From well known formula for sum of geometric series we can write:

$$
\left(\sum_{i=1}^{k} 2^{i-1}\right)=2^{k}-1 \text { and }\left(\sum_{i=1}^{k-1} 2^{i-1}\right)=2^{k-1}-1 .
$$

Table 1: Degrees, frequencies, and non-adjacencies of the representative vertices of $D_{1}[k]$

\begin{tabular}{lccc}
\hline Representative & Degree & Frequency & Non-adjacency \\
\hline$u$ & 2 & 3 & $2^{k}(18)-15$ \\
$v$ & 3 & 3 & $2^{k}(18)-16$ \\
$a_{i}$ & 3 & $3 \times 2^{i-1}$ & $2^{k}(18)-16$ \\
$b_{i}$ & 2 & $6 \times 2^{i-1}$ & $2^{k}(18)-15$ \\
$c_{i}(i \neq k)$ & 3 & $6 \times 2^{i-1}$ & $2^{k}(18)-16$ \\
$c_{n}$ & 2 & $6 \times 2^{k-1}$ & $2^{k}(18)-15$ \\
$d_{i}$ & 2 & $3 \times 2^{i-1}$ & $2^{k}(18)-15$ \\
\hline
\end{tabular}

Using Eq. 11 in Eq. 10 and simplifying, we obtain:

$$
\begin{aligned}
\bar{F}\left(D_{1}[k]\right)= & 4 \cdot\left(2^{k}(18)-15\right) \cdot\left(3+9 \cdot\left(2^{k}-1\right)+3 \cdot 2^{k}\right) \\
& +9 \cdot\left(2^{k}(18)-16\right)\left(3+3 \cdot\left(2^{k}-1\right)+6 \cdot\left(2^{k-1}-1\right)\right) .
\end{aligned}
$$

Equation 12 may be written as:

$\bar{F}\left(D_{1}[k]\right)=24 \cdot\left(2^{k}(18)-15\right)\left(2^{k+1}-1\right)+54 \cdot\left(2^{k}(18)-16\right)\left(2^{k}-1\right)$.

Further simplification of the above equation gives:

$$
\bar{F}\left(D_{1}[k]\right)=1836\left(4^{k}\right)-2988\left(2^{k}\right)+1224 .
$$

\subsection{F-coindex of type-II nanostar dendrimers $\left(D_{2}[k]\right)$}

The structure of $D_{2}[k]$ or type-II nanostar dendrimers is given in Figure 2. In case of $D_{2}[k], 10\left(2^{k+1}\right)+4\left(2^{k+2}\right)-44$ and $140\left(2^{k}\right)-127$ represent order and size, respectively.

\section{Theorem 3.2}

The F-coindex of nanostar dendrimers $\mathrm{D}_{2}[\mathrm{k}]$ is denoted by $\overline{\mathrm{F}}\left(\mathrm{D}_{2}[\mathrm{k}]\right)$ and is given as follows:

$$
\bar{F}\left(D_{2}[k]\right)=-7344 \cdot 4^{k}-18680 \cdot 2^{k}+11812, \quad \text { for } k \geq 1
$$

Proof. From Figure 2 we can analyze that expansion of $D_{2}[k]$ follows a symmetrical pattern. Therefore, for the

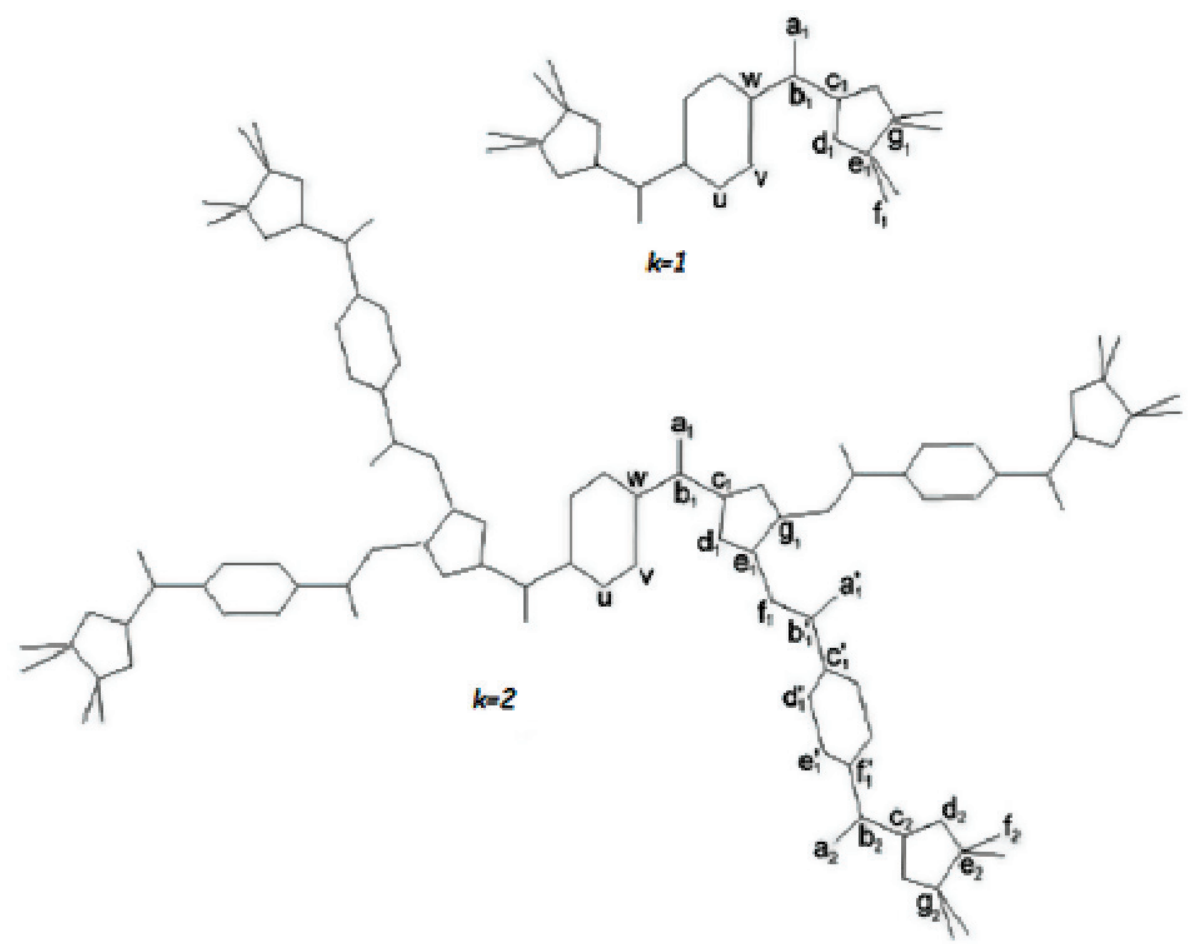

Figure 2: $D_{2}[k]$ nanostar dendrimers for $k=1$ and 2 . 
computation of F-coindex for $D_{2}[k]$, labeling of a single branch of $D_{2}[k]$ may be used. Following the symmetry of the structure three representatives namely $u, v$ and $w$, are needed for central hexagon. The remaining representatives are divided into two groups. Representatives of first group are labeled as $a_{i}, b_{i}, c_{i}, d_{i}, e_{i}$ and $f_{i}$ for $1 \leq i \leq k$. The second group of representatives for $1 \leq i_{i}^{\prime} \leq k-1$ are denoted with the symbols $a_{i}^{\prime}, b_{i}^{\prime}, c_{i}{ }_{i}, d^{\prime}{ }_{i}, e_{i}^{\prime}, f_{i}^{\prime}$. The information required for computation of F-coindex is given in Tables 2 and 3. Note that the order of $\mathrm{D}_{2}[1]$ is 28 and:

$$
N_{u}=27-d_{u} \quad \text { for } \quad u \in D_{2}[1] .
$$

Now with the help of Table 2 and formula given by Eq. 7 we can write the F-coindex of $D_{2}[1]$ as follows:

$$
\begin{aligned}
\bar{F}\left(D_{2}[1]\right)= & 1^{2} \cdot 26(2+8)+2^{2} \cdot 25(2+2+4) \\
& +3^{2} \cdot 24(2+2+2)+4^{2} \cdot 23(4)=3828 .
\end{aligned}
$$

In order to calculate $\bar{F}\left(D_{2}[k]\right)$ for $k \geq 2$, we have

We have to use the data from both tables (Tables 2 and 3). Hence the F-coindex of $D_{2}[k]$ for $k \geq 2$ can be written as:

$$
\begin{aligned}
\bar{F}\left(D_{2}[k]\right) & =1^{2} \cdot\left(36 \cdot 2^{k}-46\right)\left(\sum_{i=1}^{k} 2^{i}+2^{k+2}+\sum_{i=1}^{k-1} 2^{i+1}\right) \\
& +2^{2} \cdot\left(36 \cdot 2^{k}-47\right)\left(4+\sum_{i=1}^{k} 2^{i+1}+\sum_{i=1}^{k-1} 2^{i+1}+2 \sum_{i=1}^{k-1} 2^{i+2}\right) \\
& +3^{2} \cdot\left(36 \cdot 2^{k}-48\right)\left(2+2 \sum_{i=1}^{k} 2^{i}+4 \sum_{i=1}^{k-1} 2^{i+1}\right) \\
& +4^{2} \cdot\left(36 \cdot 2^{k}-49\right)\left(2^{k+1}\right) .
\end{aligned}
$$

Using following geometric series sum:

$$
\sum_{i=1}^{k} 2^{i+p}=2^{p+1}\left(2^{k}-1\right), \quad \text { for any positive integer } p,
$$

in Eq. 14 and simplifying, we obtain:

$$
\begin{aligned}
\bar{F}\left(D_{2}[k]\right)= & \left(36 \cdot 2^{k}-46\right)\left(8 \cdot 2^{k}-6\right)+4 \cdot\left(36 \cdot 2^{k}-47\right)\left(14 \cdot 2^{k}-20\right) \\
& +9 \cdot\left(36 \cdot 2^{k}-48\right)\left(12 \cdot 2^{k}-18\right)+32 \cdot\left(36 \cdot 2^{k}-49\right)\left(2^{k}\right) \\
& =36 \cdot 4^{k}(204)-36 \cdot 2^{k}(248)-2^{k}(9752)+11812,
\end{aligned}
$$

after further simplification of above equation, we obtain the F-coindex of $D_{2}[k]$ in the following form:

$$
\bar{F}\left(D_{2}[k]\right)=7344 \cdot 4^{k}-18680 \cdot 2^{k}+11812 .
$$

\subsection{F-coindex of poly(propyl) ether imine (PETIM) dendrimer}

We now compute the F-coindex of polynomial of poly(propyl) ether imine (PETIM) dendrimer in this
Table 2: Degrees, frequencies, and non-adjacencies of the representative vertices of $D_{2}[k]$, for $1 \leq i \leq k$

\begin{tabular}{lccc}
\hline Representative & Degree & Frequency & Non-adjacency \\
\hline$u$ & 2 & 2 & $36 \cdot 2^{k}-47$ \\
$v$ & 2 & 2 & $36 \cdot 2^{k}-47$ \\
$w$ & 3 & 2 & $36 \cdot 2^{k}-48$ \\
$a_{i}$ & 1 & $2^{i}$ & $36 \cdot 2^{k}-46$ \\
$b_{i}$ & 3 & $2^{i}$ & $36 \cdot 2^{k}-48$ \\
$c_{i}$ & 3 & $2^{i}$ & $36 \cdot 2^{k}-48$ \\
$d_{i}$ & 2 & $2^{i+1}$ & $36 \cdot 2^{k}-47$ \\
$e_{i}(i \neq k)$ & 3 & $2^{i+1}$ & $36 \cdot 2^{k}-48$ \\
$e_{k}$ & 4 & $2^{k+1}$ & $36 \cdot 2^{k}-49$ \\
$f_{i}(i \neq k)$ & 2 & $2^{i+1}$ & $36 \cdot 2^{k}-47$ \\
$f_{k}$ & 1 & $2^{k+2}$ & $36 \cdot 2^{k}-46$ \\
\hline
\end{tabular}

Table 3: Degrees, frequencies, and non-adjacencies of the representative vertices of $D_{2}[k], 1 \leq i \leq k-1$, and $k \geq 2$

\begin{tabular}{lccc}
\hline Representative & Degree & Frequency & Non-adjacency \\
\hline$a^{\prime}{ }_{i}$ & 1 & $2^{i+1}$ & $36 \cdot 2^{k}-46$ \\
$b^{\prime}{ }_{i}$ & 3 & $2^{i+1}$ & $36 \cdot 2^{k}-48$ \\
$c^{\prime}{ }_{i}$ & 3 & $2^{i+1}$ & $36 \cdot 2^{k}-48$ \\
$d^{\prime}{ }_{i}$ & 2 & $2^{i+2}$ & $36 \cdot 2^{k}-47$ \\
$e^{\prime}{ }_{i}$ & 2 & $2^{i+2}$ & $36 \cdot 2^{k}-47$ \\
$f^{\prime}$ & 3 & $2^{i+1}$ & $36 \cdot 2^{k}-48$ \\
\hline
\end{tabular}

section. We denote PETIM dendrimer by $\mathbb{G}[k]$ where $k$ represents the $k^{\text {th }}$ growth stages of $\mathbb{G}[k]$. The structure of $\mathbb{G}[k]$ for $k \geq 1$ is shown in Figure 3. It is a tree with order and size given by $24 \times 2^{k}-23$ and $24 \times 2^{k}-24$, respectively. More detail about $\mathbb{G}[k]$ may be found in Bashir et al. (2017).

To compute F-coindex for the $\mathbb{G}[k]$ shown in Figure 3, we have to use Table 4 that contains information about degree, frequency and non-adjacency of the vertices of $\mathbb{G}[k]$.

\section{Theorem 3.3}

Let $\mathbb{G}[k]$ be the molecular structure of PETIM dendrimer. Then F-coindex of $\mathbb{G}[k]$ denoted by $\overline{\mathrm{F}}(\mathbb{G}[\mathrm{k}])$ and is given by:

$$
\bar{F}(\mathbb{G}[k])=2400 \cdot 4^{k}-5064 \cdot 2^{k}+2670 .
$$

Proof. Let $\mathbb{G}[k]$ be the molecular structure of PETIM dendrimer. Depending upon the structure of $\mathbb{G}[k]$, edge set $V(\mathbb{G}[k])$ may be explained with the help of three representatives, namely $a, b$ and $c$. This division is done on the basis of degree of vertices. 


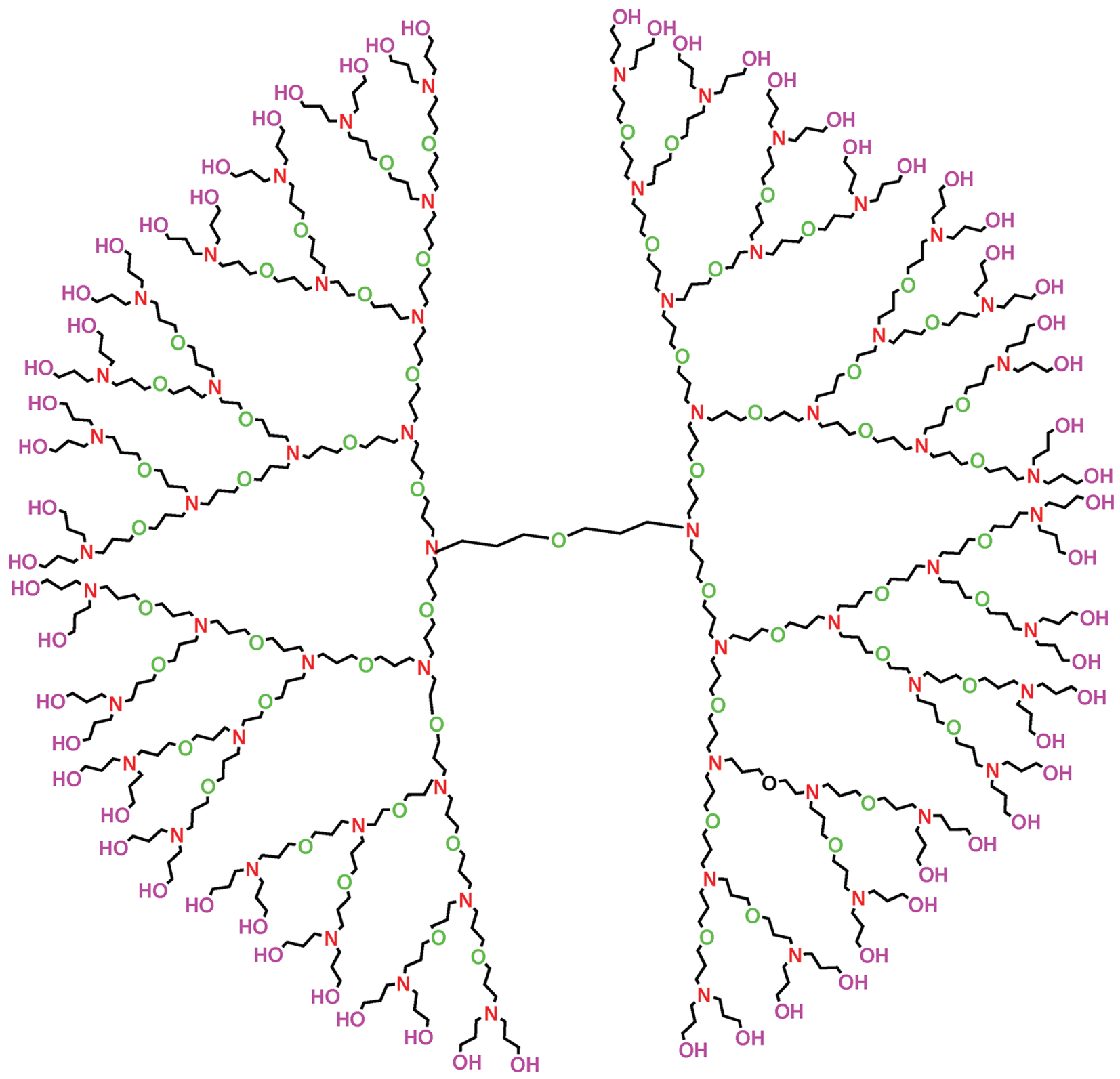

Figure 3: Growth of poly(propyl) ether imine (PETIM) dendrimer for stage $k=5$.

The degrees of $a, b$, and $c$ are 1,2 , and 3, respectively. Note that:

$$
N_{u}=24\left(2^{k}\right)-24-d_{u}, \quad \text { for } \quad u \in \mathbb{G}[k]
$$

By using the formula given in Eq. 5 and data given in Table 4, F-coindex of $\mathbb{G}[k]$ is obtained as follows:

$$
\begin{aligned}
\bar{F}(\mathbb{G}[k])= & 1^{2} \cdot\left(24 \cdot 2^{k}-25\right) \cdot 2^{k+1}+2^{2} \cdot\left(24 \cdot 2^{k}-26\right) \cdot\left(20 \cdot 2^{k}-21\right) \\
& +3^{2} \cdot\left(24 \cdot 2^{k}-27\right) \cdot\left(2^{k+1}-2\right)
\end{aligned}
$$

Simplifying the above equation gives $\bar{F}(\mathbb{G}[k])=2400 \cdot 4^{k}-5064 \cdot 2^{k}+2670$, which is required.
Table 4: Degree, frequencies, and non adjacencies of $\mathbb{G}[k]$ for $k \geq 1$

\begin{tabular}{lccc}
\hline Representative & Degree & Frequency & Non-adjacency \\
\hline$a$ & 1 & $2^{k+1}$ & $24\left(2^{k}\right)-25$ \\
$b$ & 2 & $20\left(2^{k}\right)-21$ & $24\left(2^{k}\right)-26$ \\
$c$ & 3 & $2^{k+1}-2$ & $24\left(2^{k}\right)-27$ \\
\hline
\end{tabular}

\subsection{The F-coindex of porphyrin dendrimers}

The class of porphyrin dendrimers is shown in Figures 4 and 5, for growth stages 4 and 16, respectively. It is important to note that porphyrin dendrimers are mathematically represented by $D_{k} P_{k}$, where $k=2^{m}$ for 


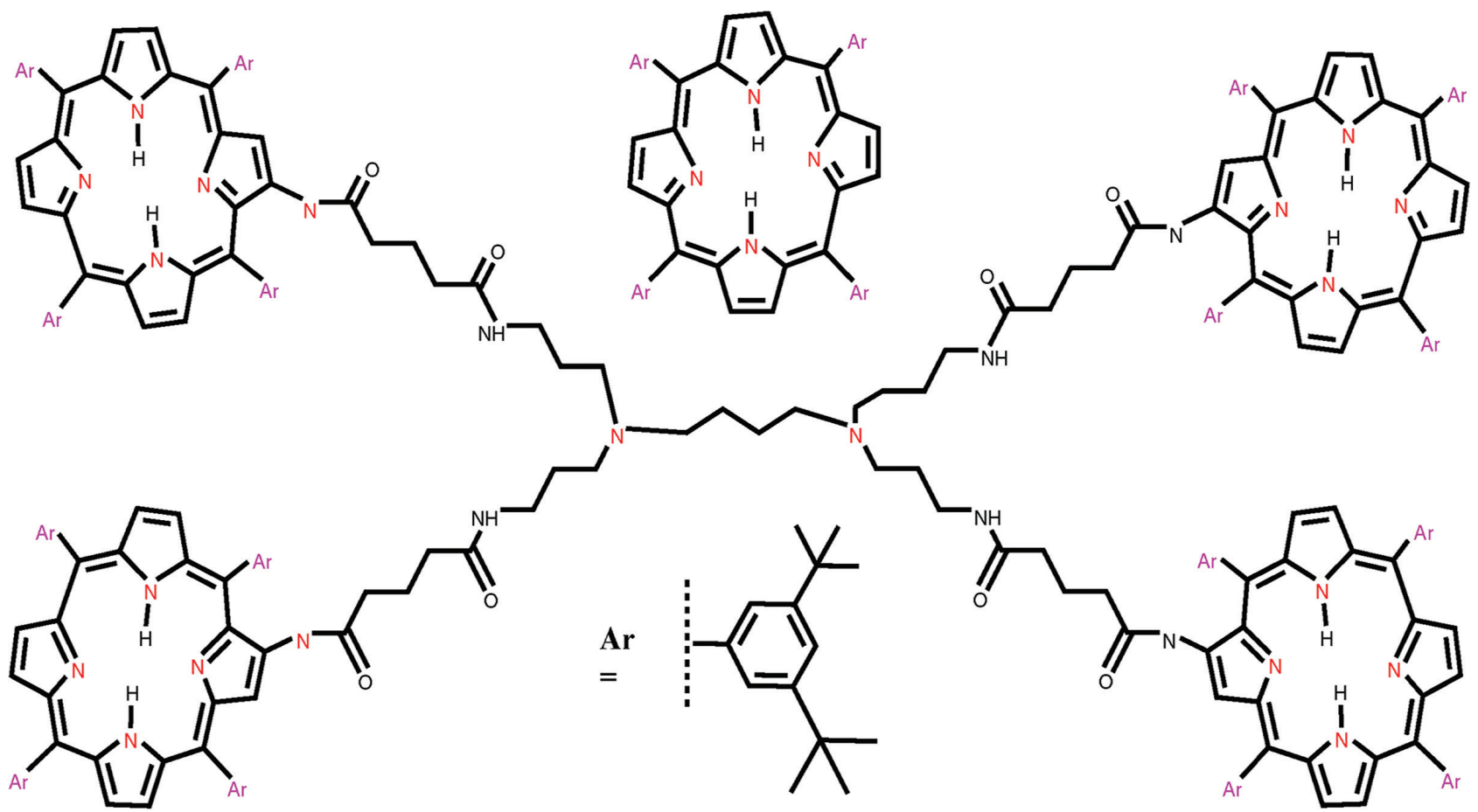

Figure 4: Molecular structure of porphyrin dendrimer $D_{4} P_{4}$.

$m \geq 2$. For the computation of forgotten descriptor for $D_{k} P_{k}$ see Bashir et al. (2017). Here we present the F-coindex for Porphyrin dendrimers. The order of $D_{k} P_{k}$ is given by $96 k-10$, where as its size is $105 k-11$. Based upon the structure of $D_{k} P_{k}$ we need four representatives. These four representatives and required information for the computation of F-coindex of $D_{k} P_{k}$ are given in the Table 5.

\section{Theorem 3.4}

Let $\mathrm{D}_{\mathrm{k}} \mathrm{P}_{\mathrm{k}}$ be a porphyrin dendrimer, then F-coindex of $\mathrm{D}_{\mathrm{k}} \mathrm{P}_{\mathrm{k}}$ denoted by $\overline{\mathrm{F}}\left(\mathrm{D}_{\mathrm{k}} \mathrm{P}_{\mathrm{k}}\right)$ and is given by:

$$
\bar{F}\left(D_{k} P_{k}\right)=52032 k^{2}-12328 k+668 .
$$

Proof. Let $D_{k} P_{k}$ be a porphyrin dendrimer, four representatives $v, w, x$, and $y$ of $D_{k} P_{k}$ and their degrees, frequencies and non-adjacencies are given in the Table 5 . Using Eq. 5 and Table 5 we have:

$$
\begin{aligned}
\bar{F}\left(D_{k} P_{k}\right)= & \left(1^{2} \cdot(96 k-12) \cdot 26 k\right)+\left(2^{2} \cdot(34 k-8) \cdot(96 k-13)\right) \\
& +\left(3^{2} \cdot(28 k-2) \cdot(96 k-14)\right)+\left(4^{2} \cdot(96 k-14) \cdot 8 k\right) .
\end{aligned}
$$

On simplifying the above equation we obtain the required result, that is,

$$
\bar{F}\left(D_{k} P_{k}\right)=52032 k^{2}-12328 k+668 .
$$

\subsection{The F-coindex of zinc-porphyrin dendrimer}

The class of dendrimer zinc-porphyrin is denoted by $D P Z_{k}$ and is shown in Figure 6. Representative vertices and their degrees, frequencies and non-adjacencies are given in the Table 6.

\section{Theorem 3.5}

Let $\mathrm{DPZ}_{\mathrm{k}}$ be a zinc-porphyrin dendrimer, then F-coindex of $\mathrm{DPZ}_{\mathrm{k}}$ denoted by $\overline{\mathrm{F}}\left(\mathrm{DPZ}_{\mathrm{k}}\right)$ and is given by:

$$
\bar{F}\left(D P Z_{k}\right)=15904\left(2^{2 k}\right)-348\left(2^{k}\right)-108 .
$$

Proof. Let $D P Z_{k}$ be a zinc-porphyrin dendrimer, representative vertices $e, f, g$ depending upon degrees are given in the Table 6. Using Eq. 7 and the Table 6 we have:

$$
\begin{aligned}
\bar{F}\left(D P Z_{k}\right)= & 2^{2} \cdot\left(44 \cdot 2^{k}-12\right) \cdot\left(56 \cdot 2^{k}-10\right) \\
& +3^{2} \cdot\left(12 \cdot 2^{k}+4\right) \cdot\left(56 \cdot 2^{k}-11\right)+4^{2} \cdot\left(56 \cdot 2^{k}-12\right),
\end{aligned}
$$

simplification gives us following expression $\bar{F}\left(D P Z_{k}\right)=15904\left(2^{2 k}\right)-348\left(2^{k}\right)-108$. 


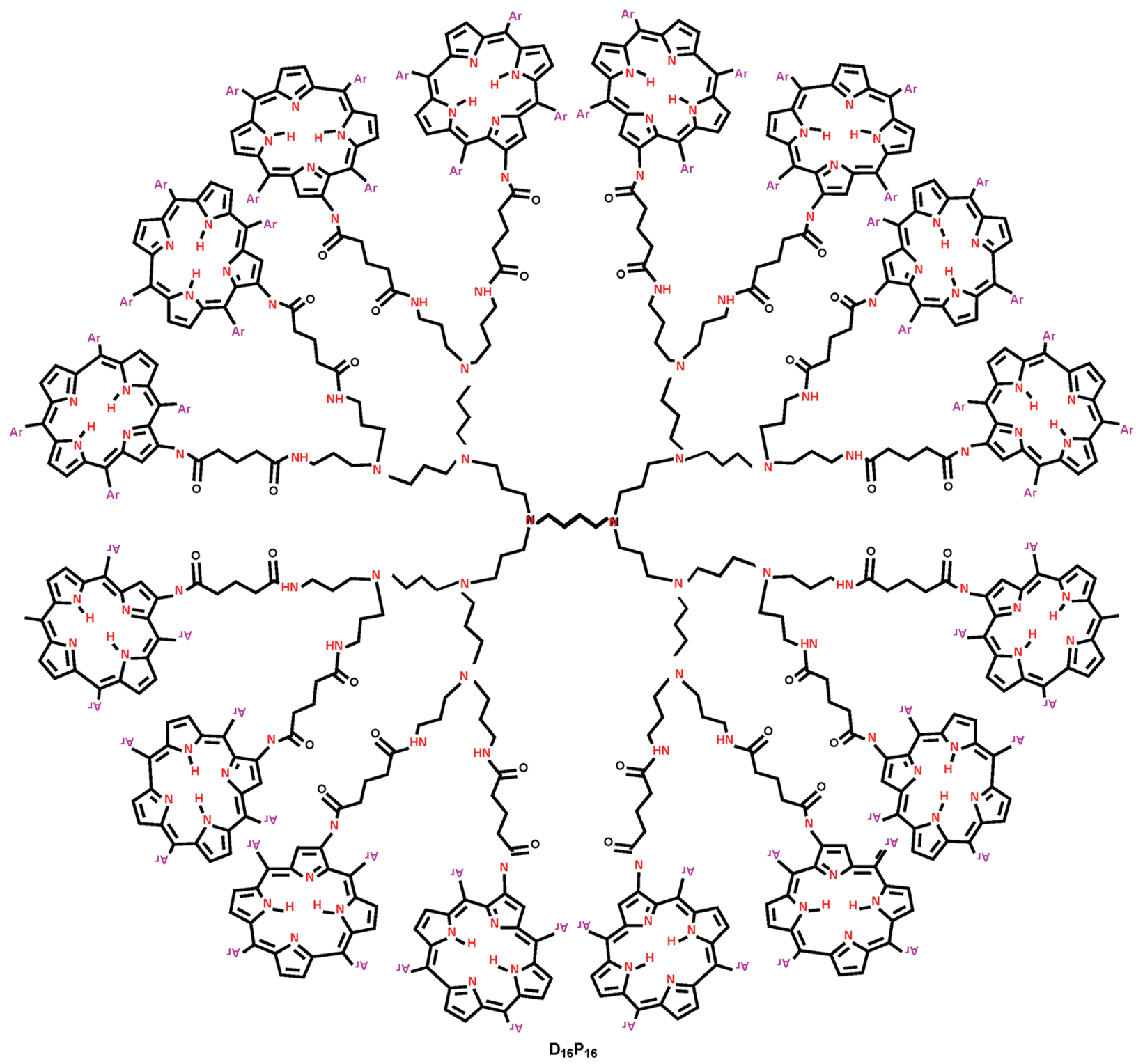

Figure 5: Molecular structure of porphyrin dendrimer $D_{16} P_{16}$.

Table 5: Degrees, frequencies, and non-adjacencies for the representatives in $D_{k} P_{k}$

\begin{tabular}{lccc}
\hline Representative & Degree & Frequency & Non-adjacency \\
\hline$v$ & 1 & $26 k$ & $96 k-12$ \\
$w$ & 2 & $34 k-8$ & $96 k-13$ \\
$x$ & 3 & $28 k-2$ & $96 k-14$ \\
$y$ & 4 & $8 k$ & $96 k-15$ \\
\hline
\end{tabular}

\section{Conclusions}

To deal with novel diseases, due to continuous growth of viruses, the development of new drugs is of prime significance. To test and predict chemical properties of these new drugs concept of topological indices is very useful especially for developing countries. In this paper, we achieved the forgotten coindex of dendrimers that are very vital for targeted delivery for cancer therapy. These results may be used in material engineering, pharmaceutical and chemical industries.

Acknowledgement: The authors are thankful to the referees for their valuable remarks. The authors would like to thank National University of Sciences and Technology (NUST) for funding this work.

Research funding: National University of Sciences and Technology (NUST). 


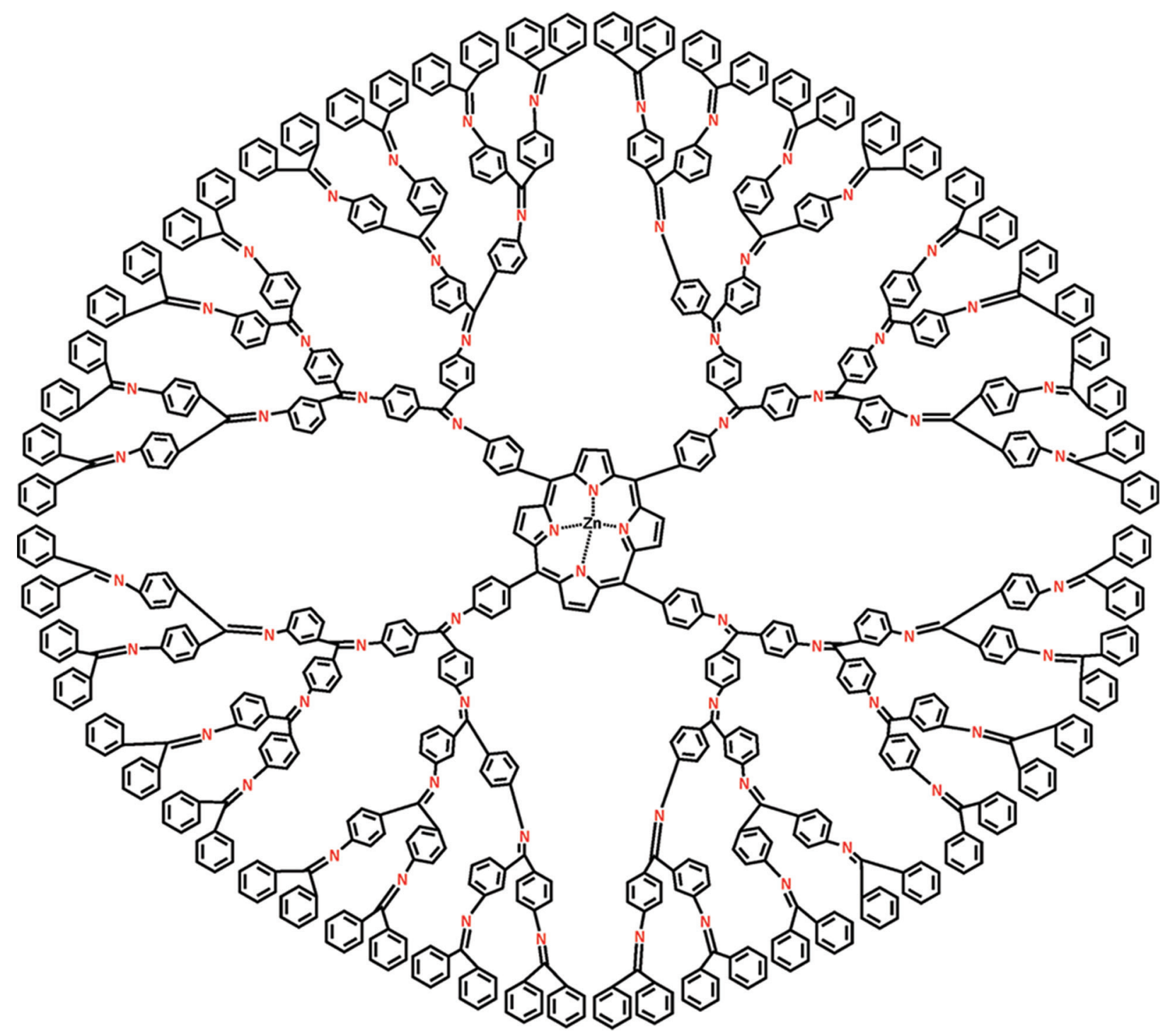

Figure 6: Molecular structure of dendrimer zinc porphyrin $D P Z_{4}$.

Table 6: Degrees, frequencies, and non-adjacencies for the representatives in $D P Z_{k}$ for $k \geq 1$

\begin{tabular}{lccc}
\hline Representative & Degree & Frequency & Non-adjacency \\
\hline$e$ & 2 & $44 \times 2^{k}-12$ & $56\left(2^{k}\right)-10$ \\
$f$ & 3 & $12 \times 2^{k}+4$ & $56\left(2^{k}\right)-11$ \\
$g$ & 4 & 1 & $56\left(2^{k}\right)-12$ \\
\hline
\end{tabular}

Author contribution: Yasir Ali: conceived of the presented idea, writing - review and editing, methodology, formal analysis; Zainab Bibi: conceived of the presented idea, writing - original draft; Quanita Kiran: writing - review and editing, methodology, formal analysis.

Conflict of interest: Authors state no conflict of interest.

\section{References}

Amin R., Abu Nayeem S.M., Trees with minimum F-coindex. Electron. Notes Discret. Math., 2017, 63, 303-310.

Bielinska A.U., Chen C., Johson J., Baker Jr. J.R., DNA complexing with polyamidoamine dendrimers: implications for transfection. Bioconjugate Chem., 1999, 10(5), 843-850.

Bashir Y., Aslam A., Kamran M., Qureshi M.I., Jahangir A., Rafiq M., et al., On forgotten topological indices of some dendrimers structure. Molecules, 2017, 22(6), 867.

Berhe M., Wang C., Computation of certain topological coindices of graphene sheet and $\mathrm{C} 4 \mathrm{C} 8(\mathrm{~S})$ nanotubes and nanotorus. Appl. Math. Nonlin. Sci., 2019, 4(2), 455-468.

De N., F-index and coindex of some derived graphs. Bull. Inter. Math. Virtual Inst., 2018, 8, 81-88.

De N., Abu Nayeem S.M., Computing the F-index of nanostar dendrimers. Pac. Sci. Rev. A-Nat. Sci. Eng., 2016, 18, 14-21. 
De N., Abu Nayeem S.M., Pal A., The F-coindex of some graph operations. Springer Plus, 2016, 5.

Diudea M.V., Vizitiu A.E., Mirzagar M., Ashrafi A.R., Sadhana polynomial in nano-dendrimers. Carpathian J. Math., 2010, 26(1), 59-66.

Estrada E., Atom-bond connectivity and the energetic of branched alkanes. Chem. Phys. Lett., 2008, 463, 422-425.

Estrada E., Torres L., Rodriguez L., Gutman I., An atom-bond connectivity index: Modelling the enthalpy of formation of alkanes. Indian J. Chem., 1998, 37A, 849-855.

Ethirajan M., Chen Y., Joshi P. Pandey R.K., The role of porphyrin chemistry in tumor imaging and photodynamic. therapy. Chem. Soc. Rev., 2011, 40, 340-362.

Furtula B., Gutman I., A forgotten topological index. J. Math. Chem., 2015, 53, 1184-1190.

Gao W., Farahani M.R., Shi L., Forgotten topological index of some drug structures. Acta Med. Medit., 2016, 32, 579-585.

Gao W., Akhter S., Iqbal Z., Qasim M., Aslam A., The topological aspects of phthalocyanines and porphyrins dendrimers. IEEE Access, 2020a, 8, 168631-168649.

Gao W., Butt S.I., Numan M., Aslam A., MalikZ., Waqas M., Omega and the related counting polynomials of some chemical structures. Open Chem., 2020b, 18(1), 1167-1172.

Gao W., Guirao J.L.G., Wu H., Nordhaus-Gaddum type inequalities for some distance-based indices of bipartite molecular graphs. J. Math. Chem., 2020c, 58(7), 1345-1352.

Gonzaga V.R., da Silva Santos S., da Silva J.V., Campos Prieto D., Feliciano Savino D., Giarolla J., et al., Targeting groups employed in selective dendrons and dendrimers. Pharmaceutics, 2018, 10(4), 219.

Guo C., Zhang Y., Sun M., Wang Z., Fan A., Zhao Y., Modulating topical drug delivery via skin pre-treatment with low-generation poly(amidoamine) dendrimers. J. Drug Deliv. Sci. Tec., 2014, 24, 555.

Gutman I., Degree-based topological indices. Croat. Chem. Acta, 2013, 86, 351-361.

Gutman I., Tosović J., Radenković S., Marković S., On atom-bond connectivity index and its chemical applicability. Indian J. Chem., 2012, 51A, 690-694.

Gutman I., Trinajstić N., Graph theory and molecular orbitals. Total -electron energy of alternant hydrocarbons. Chem. Phys. Lett., 1972, 17, 535-538.

Imran M., Hayat S., Mailk M.Y.H., On topological indices of certain interconnection networks. Appl. Math. Comput., 2014, 244, 936-951.

Jain S., Kaur A., Puri R., Utreja P., Jain A., Bhide M., et al., Poly propyl ether imine (PETIM) dendrimer: A novel non-toxic dendrimer for sustained drug delivery. Eur. J. Med. Chem., 2010, 45(11), 4997-5005.

Jeong Y., Yoon H. Jang W., Dendrimer porphyrin-based self-assembled nano-devices for biomedical applications. Polym. J., 2012, 44, 512-521.

Lakshminarayanan A., Ravi V.K., Tatineni R., Rajesh Y.B.R.D., Maingi V., Vasu K.S., et al., Efficient dendrimer-DNA complexation and gene delivery vector properties of nitrogen-core poly(propyl ether imine) dendrimer in mammalian cells. Bioconjugate Chem., 2013, 24(9), 1612-1623.

Lee S.J., Jeong Y.-I., Park H.-K., Kang D.W., Oh J.-S., Lee S.-G., et al., Enzyme-responsive doxorubicin release from dendrimer nanoparticles for anticancer drug delivery. Int. J Nanomedicine, 2015, 10, 5489-5503.

Liu J., Ali B., Malik M.A., Siddiqui H.M.A., Imran M., Reformulated Zagreb indices of some derived. Mathematics, 2019, 7, 283.

Mitragotri S., Burke P.A., Langer R., Overcoming the challenges in administering biopharmaceuticals: formulation and delivery strategies. Nat. Rev. Drug Discov., 2014, 13(9), 655-672.

O'Connor A. E., Byrne A.T., Gallagher W.M., Porphyrin and non-porphyrin photosensitizers in oncology: pre-clinical and clinical advances in photodynamic therapy. Photochem. Photobiol., 2009, 85, 1053-1074.

Sadamoto R., Tomioka N. Aida T., Photoinduced electron transfer reactions through dendrimer architecture. J. Am. Chem. Soc., 1996, 118, 3978-3979.

Shao Z., Siddiqui M.K., Muhammad M.H., Computing Zagreb indices and Zagreb polynomials for symmetrical nanotubes. Symmetry, 2018, 10, 244.

Siddiqui H.M.A., Computation of Zagreb indices and Zagreb polynomials of Sierpinski graphs. Hacet. J. Math. Stat., 2020, 49(2), 754-765.

Sun M., Fan A., Wang Z., Zhao Y., Dendrimer-mediated drug delivery to the skin. Soft Matter, 2012, 8, 4301-4305.

Sun Y.C., Lin Z., Peng W.X., Yuan T.Q., Xu F., Wu Y.Q., et al., Chemical changes of raw materials and manufactured binderless boards during hot pressing: lignin isolation and characterization. Bioresources, 2014, 9(1), 1055-1071.

Xiong Z., Shen M., Shi X., Dendrimer-based strategies for cancer therapy: Recent advances and future perspectives. Sci. China Mater., 2018, 61(11), 1387-1403.

Xu J., Liu J., Bilal A., Ahmad U., Siddiqui H.M.A., Ali B., et al., Distance degree index of some derived graphs. Mathematics, 2019, 7, 283.

Yang H., Targeted nanosystems: Advances in targeted dendrimers for cancer therapy. Nanomed.-Nanotechnol., 2016, 12,2, 309-316.

Yang J., Zhang Q., Chang H., Cheng Y., Surface-engineered dendrimers in gene delivery. CHEM REV., 2015, 115, 5274-5300. 